\title{
HM89 Regimen
}

National Cancer Institute

\section{Source}

National Cancer Institute. HM89 Regimen. NCI Thesaurus. Code C67212.

A regimen administered in four phases that is used for the treatment of disseminated, childhood, anaplastic large cell lymphoma (ALCL). The 4 phases in the regimen include: COP (cyclophosphamide, vincristine and prednisone); COPADM (cyclophosphamide, vincristine, prednisone, doxorubicin, and methotrexate); VEM (etoposide, cyclophosphamide and methotrexate); and VAD (vincristine and doxorubicin). 